\title{
Call admission control based on resource sharing in LTE-Advanced relay system
}

\author{
Han Haifang ${ }^{\mathrm{a}}$, Zhao Jihong ${ }^{\mathrm{ab}}$ \\ a. Xi'an University of Posts \& Telecommunications, Xi'an 710061, China \\ b. Nanjing University of Posts \& Telecommunications, Nanjing 210003, China; \\ han_haifang@163.com,eeleeg@gmail.com
}

\begin{abstract}
We have proposed a call admission control in
LTE-Advanced relay system which is based on resource sharing.

Each cell has 7 sites, including 1 eNB and 6 relay stations, if the shared resources are used up for handover users and new users in the requested site, handover users can use the reserved resources of the requested site, while new users can utilize the resources of other sites in the same cell. Simulation results show that our proposed algorithm can effectively reduce the dropping rate of handover users and blocking rate of new users when compared with the conventional ones and, in the meantime, improve the system resource utilization.
\end{abstract}

Key words-LTE-A; call admission control; resource sharing; relay

\section{I . INTRODUCTION}

The 3GPP (the 3rd Generation Partnership Project) have pointed out that call admission control is to make a judgment of whether to accept a new radio bearer request [1]. With the development of communication technology, users are increasingly demanding high QoS (quality of service), and, at the same time, users are more sensitive to the QoS. Users want to be accessed to the network quickly whenever and wherever they are and enjoy low-latency, high-speed, seamless switching handover services, which requires the mobile communication system to provide the corresponding QoS assurance [2]. However, the radio resource in mobile communication system is limited, if too many users are accessing the system, the individual QoS will be deteriorated, so the reasonable call admission control method is the key to alleviate the contradiction between the limited radio resource and the high individual QoS. At present, the main strategies of call admission control are completely sharing strategy, fully differentiate strategy, adaptive bandwidth reservation scheme and fixed bandwidth reservation scheme [3]. These strategies can reduce the dropping rate of handover users and blocking rate of new users to a certain extent, but they cannot fully meet the needs of users.

With the development of wireless communication technology, CA (Carrier Aggregation), MIMO (Multi-input/Multi-output), CoMP (Coordinated Multi Point Transmission / Reception), Relaying and other new technologies are introduced in LTE-A system. The introduction of relay station can not only extend the cell coverage, but also improve the system capacity, and the relay stations can benefit the users at the cell edge [4]. The introduction of relay station makes LTE-A system evolved into LTE-A relay system, and its typical method of CAC (call admission control) is fixed bandwidth reservation scheme. The scheme is characterized by setting aside a portion of resources reserved for handover users: the handover users and new users share the shared resources in competition, while the reserved resources could only be used by the handover users. But when the new users arrive at a high rate, the system will suffer from high blocking rate for the new users due to the discrepancy between the flexible distribution of call access and rigid configuration of spectral resource, which leads to a high decline of the acceptance rate [5]. In order to avoid this phenomenon and reduce the blocking rate of new users in this paper, the CAC algorithm on fixed bandwidth reservation is evolved into on resource sharing in LTE-A relay system. In this algorithm, the user requests access into the system from a site, if the sharing resources for handover users and new users have been used up in the site, handover users can access to the system by the reserved resources for handover users of the site, while new users can access to the system by successfully resource sharing between the target site and adjacent sites within the cell. This improved method not only reduces the dropping rate of handover users and new users blocking rate, 
but also improve the resource utilization of the system compared to the convention one.

\section{II . DESCRIPTION of OUR ALGORITHM}

\section{A. System Model}

System model in this paper is showed in Figure 1:

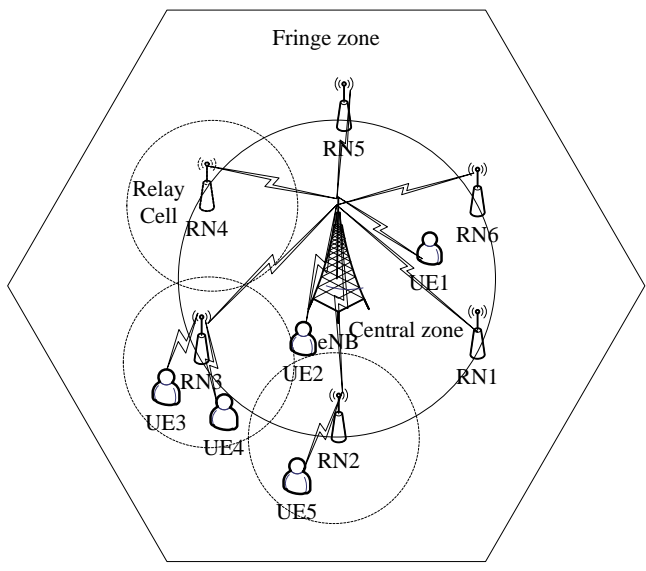

Fig.1. System model of the LTE-A relay system

In this paper, the macro-cellular layout model is composed of two-tier 19 cells, each cell has 7 sites, including 1 eNB and 6 relay stations, and the radius of the cell is R. The eNB is located in the center, while the 6 relay stations are uniformly placed on the arc $2 / 3 * \mathrm{R}$ away from the cell center, eNB and relays are all assumed to be equipped with omni-directional antennas. Since the transmit power of relay station is less than that of eNB, so the coverage of relay station is less than the coverage of eNB. We consider uplink CAC in this paper, each user selects the access site according to the received SINR level from the eNB or a relay station. ENB undertakes $40 \%$ of the whole system load, while every relay station undertakes $10 \%$ of the system load. As shown in Fig.2. and Fig.3. the entire frequency band is divided into 10 parts uniformly, the eNB is assigned 4 parts of the frequency, while the 6 relay stations are each assigned 1 parts of the remaining 6 parts of the frequency, and each cell has the same frequency allocation. $20 \%$ of the assigned frequency is reserved for handover users, while the remaining $80 \%$ frequency is used for both handover users and new users in each site. For each user, using Round Robin(RR) algorithm for resource allocation.

\begin{tabular}{|l|l|l|l|l|l|l|l|l|l|}
\hline F1 & F2 & F3 & F4 & F5 & F6 & F7 & F8 & F9 & F10 \\
\hline
\end{tabular}

Fig.2. Frequency allocation of the LTE-A relay system

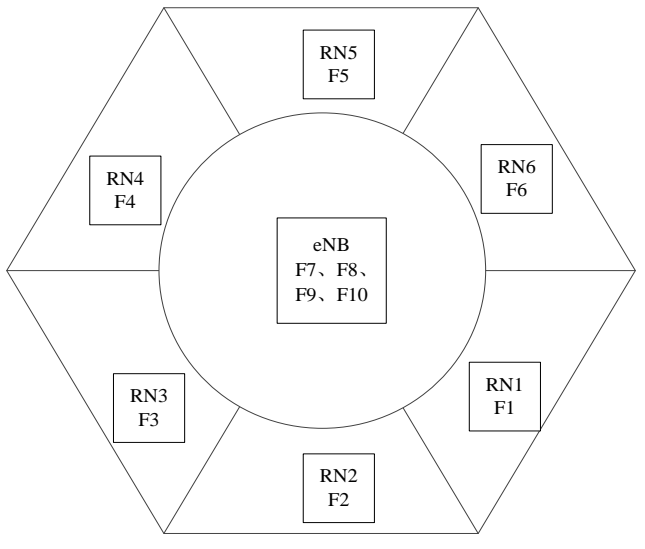

Fig.3. Frequency assignment of the LTE-A relay system

\section{B. Algorithm Description}

The literature [2] pointed out that the CAC algorithm needs to deal with two types of user-initiated connection request, one is the N-UE (new-user) - initiated connection request, and the other is the H-UE (handover user) -initiated connection request. The N-UE-initiated connection request is made by the user locates in the current cell, while the H-UE-initiated connection request is made by the user locates in the neighboring cell, which is a result of the user mobility and the user needs to switch to the current cell for continuous connection to the system and preferable QoS. Therefore, in the study of CAC algorithm, user requests only include new user connection request and handover user connection requests. As call interruption is more unacceptable than the call block, the handover call has a higher priority over the new call in the CAC algorithm. This algorithm is based on the judgment of current load conditions of the system without a prediction mechanism. This does not consider whether the access of the user's request will lead to a high load of the system. The commercial experience of WCDMA has proved that accurate prediction mechanism brings in computation complexity, not better than no prediction mechanism in final effect [7].

In this paper, two types of thresholds are set up for each site: one is the rejection threshold, and the other is the new user-paid access threshold. For each site, the new user-paid access threshold less than it's rejection threshold. Rejection threshold is set to be the largest business of the site, over which both handover users and new users would be neglected new user-paid access threshold is set to be $80 \%$ of the largest 
business of the site, over which handover users accessed only.

The flow chart of a call admission control based on resource sharing in LTE-Advanced relay system is shown in Figure 2:

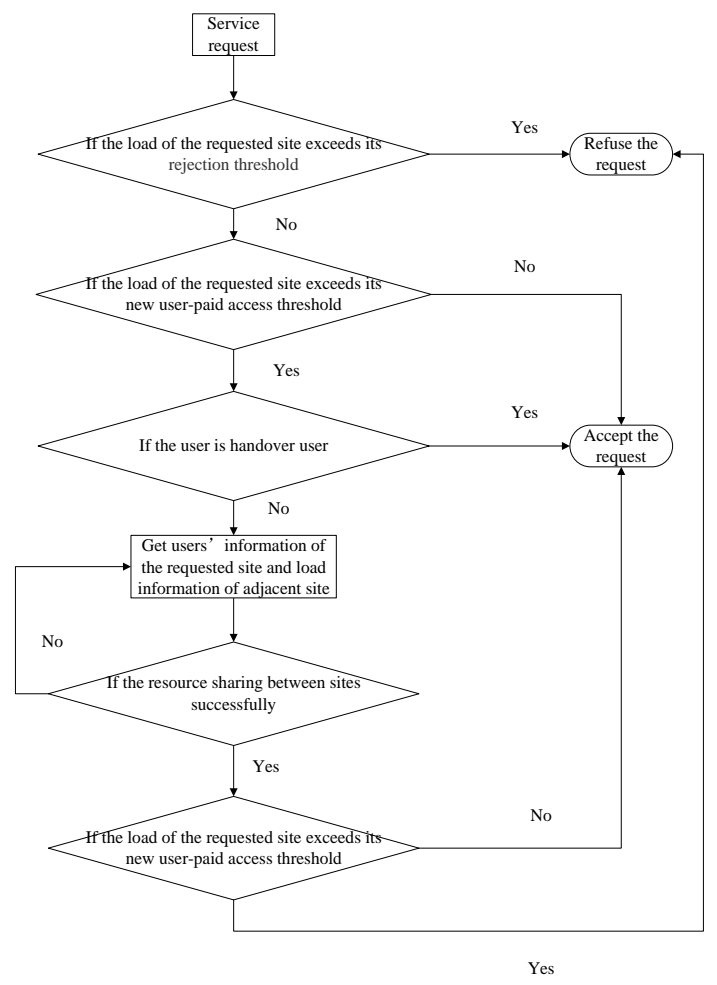

Fig.4. The flow chart of a CAC based on resource sharing in LTE-Advanced relay system

As the flow chart is shown in Fig.4, if a user requests access to the system:

1): If the load of the site is greater than or equal to the rejection threshold of the site, both new user and handover user can not access to the system;

2): If the load of the site is less than the new user-paid access threshold of the site, both new user and handover user can access to the system;

$3)$ : If the load of the site is greater than or equal to the new user-paid access threshold of the site and less than the rejection threshold of the site, handover users could be accepted, while new user need to wait until the resources between the site and adjacent site within the cell are successfully shared, and enough resources are given to the requesting new user, otherwise the new user is denied.

The resources sharing among sites as follows:

The site who shares resources with the requested site is the target site, the user who accesses to the system from via its requested site to the target site is the target user.

Resource sharing conditions are as follows:

$$
\begin{aligned}
\mathrm{L}_{\mathrm{r}}(\mathrm{A}) & \leq \mathrm{L}_{\mathrm{r}} \leq \mathrm{L}_{\mathrm{r}}(\mathrm{B}) \\
\mathrm{L}_{\mathrm{SINR}} & \leq \mathrm{SINR}_{\mathrm{t}} \\
\mathrm{L}_{\mathrm{t}} & \leq \mathrm{L}_{\mathrm{t}}(\mathrm{B})
\end{aligned}
$$

$\mathrm{L}_{\mathrm{r}}$ indicates the current load of the requested site, while $\mathrm{L}_{\mathrm{r}}(\mathrm{A})$ means the new user-paid access threshold of the requested site, $\mathrm{L}_{\mathrm{r}}(\mathrm{B})$ says the rejection threshold of the requested site at the same time, so equation (1) indicates the current load of the requested site between its new user-paid access threshold and rejection threshold. SINR $_{t}$ means the received SINR from the target site of the user who has accessed to the system via the requested site, while $\mathrm{L}_{\mathrm{SINR}}$ represents the SINR threshold to access to the system of the target user, so equation (2) indicates the SINR of the target user receives from the target site meets the target user's threshold to access to the system. $\mathrm{L}_{\mathrm{t}}$ means the current load of the target site, while $\mathrm{L}_{\mathrm{t}}(\mathrm{B})$ says the rejection threshold of the target site, so equation (3) indicates the current load of the target site is less than the rejection threshold of the target site.

Detailed CAC algorithm process is as follows :

If there is a new user A requests access to system via eNB, the load of eNB satisfies equation (1), namely the eNB's load exactly between it's new user-paid access threshold and rejection threshold. System tests whether there is a user who has accessed to the system via eNB satisfies equation (2).

First of all, if all the users who have accessed to the system via eNB don't satisfy equation (2), the system refuses to accept the new user A.

Secondly, if there is a user who has accessed to the system via eNB satisfies equation (2), but all the relay stations around eNB do not meet equation (3), system refuses to accept the new user $\mathrm{A}$.

Finally, if the SINR from $\mathrm{RN}_{\mathrm{j}}$ received by user $_{\mathrm{i}}$ who has accessed to the system via eNB meets equation (2), and $\mathrm{RN}_{\mathrm{j}}$ satisfies equation (3), so user $i$ is the target user, and $\mathrm{RN}_{\mathrm{j}}$ is the target site. The system makes user $_{i}$ accesses to the system from via eNB to via $\mathrm{RN}_{\mathrm{j}}$, and resource sharing success, while achieves resource sharing between eNB and relay station. For user $_{\mathrm{i}}$, if there are many relay stations meet equation (2) simultaneously, the relay station who's SINR is maximum received by user $_{i}$ is the target site. System detects the load 
information of eNB, if the load is less than the new user-paid access threshold of eNB, accepts the new user A, stops the detection process of judging whether the users who have accessed to the system via eNB meet equation (2) at the same time; if the load still higher than the new user-paid access threshold of eNB, system refuses to accept the new user A. Although the new user A is denied, the load of eNB is reduced in a certain extent.

There is a handover user $\mathrm{B}$ requests access to system via eNB, if the load of eNB is greater than or equal to its rejection threshold, system refuses to accept the handover user B; if the load of eNB is less than its rejection threshold, system accepts the handover user $\mathrm{B}$.

If there is a new user $C$ requests access to system via $\mathrm{RN}_{\mathrm{i}}$, the load of $\mathrm{RN}_{\mathrm{i}}$ satisfies equation (1) at this time, namely the load of $\mathrm{RN}_{\mathrm{i}}$ exactly between its new user-paid access threshold and rejection threshold. System tests whether there is a user who has accessed to the system via $\mathrm{RN}_{\mathrm{i}}$ satisfies equation (2).

First of all, if all the users who have accessed to the system via $\mathrm{RN}_{\mathrm{i}}$ don't satisfy equation (2), the system refuses to accept the new user $\mathrm{C}$.

Secondly, if there is a user who has accessed to the system via $R N_{i}$ satisfies equation (2), but the two relay station and eNB who adjacent to $\mathrm{RN}_{\mathrm{i}}$ do not meet equation (3), system refuses to accept the new user $\mathrm{C}$.

Finally, if the SINR of site $_{k}\left(\right.$ site $_{\mathrm{k}}$ can be one of the two Relay station and eNB who adjacent to $\mathrm{RN}_{\mathrm{i}}$ ) received by $\mathrm{user}_{\mathrm{j}}$ who has accessed to the system via $\mathrm{RN}_{\mathrm{i}}$ meets equation (2), and site $_{\mathrm{k}}$ satisfies equation (3), so user $_{\mathrm{j}}$ is the target user, and

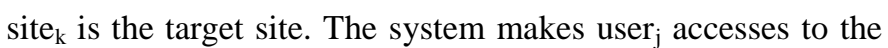
system from via $\mathrm{RN}_{\mathrm{i}}$ to via site $\mathrm{k}_{\mathrm{k}}$, and resource sharing success. If the site $_{\mathrm{k}}$ is a relay station, the resource sharing is between relay stations; if the site $_{\mathrm{k}}$ is the $\mathrm{eNB}$, the resource sharing is between relay station and eNB. For user $r_{j}$, if there are many sites meet equation (2) simultaneously, the site who's SINR is maximum received by user $_{\mathrm{j}}$ is the target site. System detects the load information of $\mathrm{RN}_{\mathrm{i}}$, if the load is less than the new user-paid access threshold of $\mathrm{RN}_{\mathrm{i}}$, accepts the new user $\mathrm{C}$, stops the detection process of judging whether the users who have accessed to the system via $\mathrm{RN}_{\mathrm{i}}$ meet equation (2) at the same time; if the load still higher than the new user-paid access threshold of $\mathrm{RN}_{\mathrm{i}}$, system refuses to accept the new user
C. Although the new user $\mathrm{C}$ is denied, the load of $\mathrm{RN}_{\mathrm{i}}$ is reduced in a certain extent.

There is a handover user $\mathrm{D}$ requests access to system via $\mathrm{RN}_{\mathrm{i}}$, if the load of $\mathrm{RN}_{\mathrm{i}}$ is greater than or equal to its rejection threshold, system refuses to accept the handover user D; if the load of $\mathrm{RN}_{\mathrm{i}}$ is less than its rejection threshold, system accepts the handover user D.

\section{SIMULATION RESULTS AND DISCUSSION}

In this paper, the macro-cellular layout model is composed of two-tier 19 cells, each cell is allocated 10M bandwidth and the number of RBs is 50. Assuming a RB only serve a user at a time, every cell can serve at most 50 users simultaneously. The eNB is assigned $20 \mathrm{RBs}$ with $4 \mathrm{RBs}$ are reserved for handover users only, while each relay stations is assigned $5 \mathrm{RBs}$ with 1 $\mathrm{RB}$ is reserved for handover users only.10000 users are randomly distributed within the cell and the ratio between the number of new users and the handover ones is $4: 1.20 \%$ of the users are selected as handover users randomly. The arrivals of both new users and handover users subject to Poisson distribution. Arrival rate of user as shown in Table I. The service process of each user submits to negative exponential distribution, and the service rate is $1 / 300$ with the average service time being 300s. According to the different arrival rates of new users and handover users, we observe the resource utilization, the blocking rate of new users and dropping rate of handover users respectively.

TABLE I. SIMULATION PARAMETERS

\begin{tabular}{|l|l|}
\hline \multicolumn{1}{|c|}{ parameters } & \multicolumn{2}{c|}{ Value } \\
\hline Inter-Site Distance & $500 \mathrm{~m}$ \\
\hline Carrier Frequency & $2000 \mathrm{MHz}$ \\
\hline \multicolumn{1}{|c|}{ Bandwidth } & $10 \mathrm{MHz}$ \\
\hline $\begin{array}{l}\text { Number of resource } \\
\text { block }\end{array}$ & 50 \\
\hline eNB Transmit Power & $46 \mathrm{dBm}$ \\
\hline RN Transmit Power & $30 \mathrm{dBm}$ \\
\hline eNB Antenna Gain & $14 \mathrm{dBi}$ \\
\hline RN Antenna Gain & $5 \mathrm{dBi}$ \\
\hline Traffic Model & Full Buffer \\
\hline $\begin{array}{l}\text { Thermal Noise Spectral } \\
\text { Density }\end{array}$ & $-174 \mathrm{dBm} / \mathrm{Hz}$ \\
\hline \multicolumn{1}{|c|}{ Path Loss } & PL=128.1+37.6log ${ }_{10}(\mathrm{R})$ \\
\hline Shadowing Standard & eNB-UE: $8 \mathrm{~dB}$ \\
\hline
\end{tabular}




\begin{tabular}{|l|l|}
\hline Deviation & RN-UE: 10dB \\
\hline SINR threshold & $5 \mathrm{~dB}$ \\
\hline Number of cell users & 10000 \\
\hline User distribution & Random distribution \\
\hline $\begin{array}{l}\text { Minimum Distance } \\
\text { between eNB and users }\end{array}$ & $35 \mathrm{~m}$ \\
\hline $\begin{array}{l}\text { Minimum Distance } \\
\text { between RN and users }\end{array}$ & $10 \mathrm{~m}$ \\
\hline $\begin{array}{c}\text { Ratio of new user and } \\
\text { handover user }\end{array}$ & $4: 1$ \\
\hline Arrival rate of user & $0.001-0.01$, step:0.001 \\
\hline User service time & $300 \mathrm{~s}$ \\
\hline
\end{tabular}

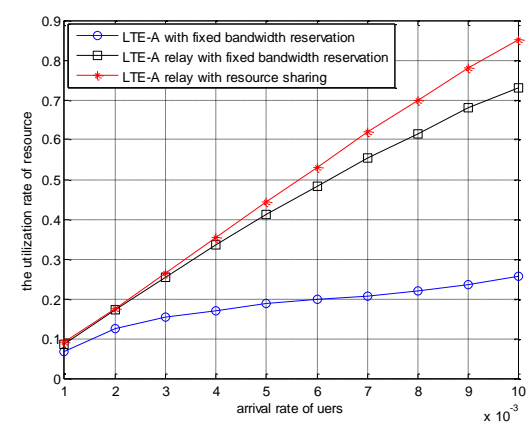

Fig.5. the resource utilization of system

LTE-A relay with resource sharing is our proposed algorithm. Fig.5 gives the resource utilization of the system, the proposed algorithm is the highest in the case of same user arrival rate. After the initial assignment of resource block for each site within one cell, a lighter load site can bear part load of a heavier load site by resource sharing within a cell to reduce load of the heavier loaded sites, improving the acceptance ability of the heavier load site at the same time.

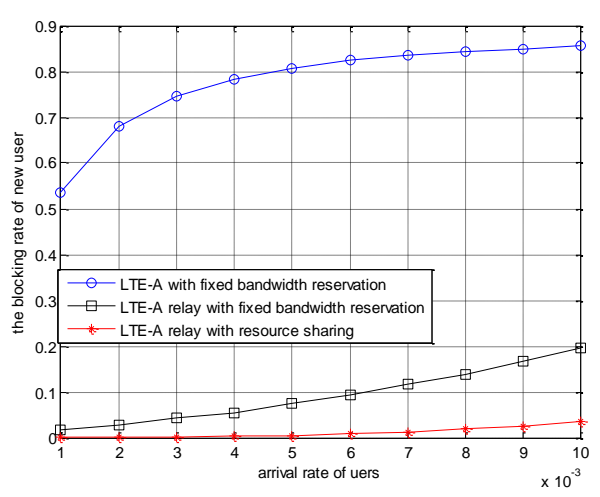

Fig.6. the blocking rate of new users
Fig.6 shows the blocking rate of new users, algorithm of LTE-A with fixed bandwidth reservation is the highest, followed by algorithm of LTE-A relay with fixed bandwidth reservation, and algorithm of LTE-A relay with resource sharing is the lowest. This is because the introduction of relay station makes the edge users of algorithm of LTE-A relay with fixed bandwidth reservation can receive a better signal quality, and improves the probability of user accessed to the system, while reduces the blocking rate of new users, thereby algorithm of LTE-A relay with fixed bandwidth reservation is lower than algorithm of LTE-A with fixed bandwidth reservation in the blocking rate of new users; if the load of the requested site of new user is higher than its new user-paid access threshold, system frees up resources for the new user by making the target user accessed to the system from the requested site to the target site through resource sharing between the requested site and target site within the cell instead of rejecting the new user. Thus the system resource utilization is improved and the blocking rate of new users is reduced, the blocking rate of new users of algorithm of LTE-A relay with resource sharing is the lowest.

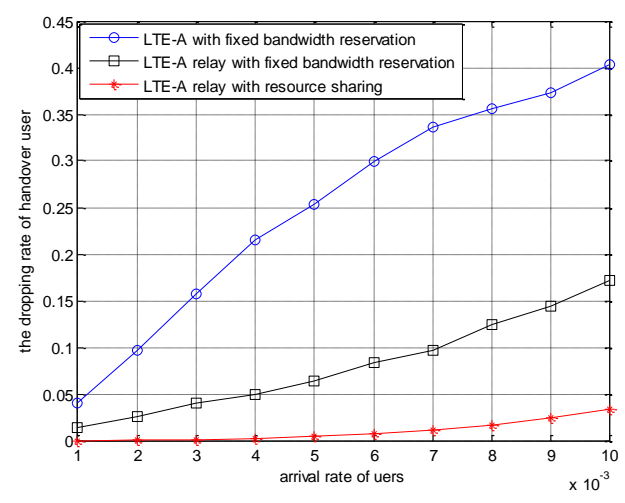

Fig.7. the dropping rate of handover users

Fig.7 gives the dropping rate of handover users, algorithm of LTE-A with fixed bandwidth reservation is the highest, followed by algorithm of LTE-A relay with fixed bandwidth reservation, and algorithm of LTE-A relay with resource sharing is the lowest. This is due to the introduction of relay station narrow the gap between the edge users of algorithm of LTE-A relay with fixed bandwidth reservation and their accessed sites, improves the probability of user accessed to the system, reduces the dropping rate of handover users, thereby algorithm of LTE-A relay with fixed bandwidth reservation is 
lower than algorithm of LTE-A with fixed bandwidth reservation in the dropping rate of handover users; since users needs to free up resources for new users by switching their accessed site from original site to target site, achieve load balancing between the 7 sites within a cell, the system resource utilization is improved, thus the dropping rate of handover users of algorithm of LTE-A relay with resource sharing is the lowest.

\section{CONCLUSIONS}

A CAC in LTE-A relay system which is based on resource sharing is proposed in this paper, it takes a different admission control scheme according to the current load of the requested site and the type of the requested user, if it is a new user or a handover one. Simulation results show that the proposed algorithm can improve the resource utilization, reducing the blocking rate of new users and the dropping rate of handover users. The proposed algorithm achieves resource sharing between eNB and relay station, relay station and relay station, relay station and eNB in a certain extent.

\section{ACKNOWLEDGMENT}

This work is supported by the National Natural Science Foundation of China(No.61371087) and the National Science and Technology Major Projects "New Generation Broadband Wireless Mobile Communication Network" (No.2012ZX03001029-003、2012ZX03001008-003)

\section{REFERENCES}

[1] Huan Xiong. Cross-layer design of admission control based on resource utilization for LTE-Advanced system [D]. Xi'an: xi'an university of post \& telecommunications, 2012

[2] Shuguang Wang. Investigation on radio admission control in LTE system [D]. Chengdu: Southwest Jiaotong University,2011

[3] Qian Y, Tipper D, Medhi D. A nonstationary analysis of bandwidth access control schemes for heterogeneous traffic in B-ISDN [C]. In: INFOCOM '96. Fifteenth Annual Joint Conference of the IEEE Computer Societies, 1996: 730-737.

[4] Ali Yaver, Damian Kolmas, Jaroslaw Lachowski. Resource Utilization with Relays in LTE-Advanced Networks. IEEE CCECE [J].2011.

[5] Yang Wang. The study of admission control algorithm in TD-LTE [D].Nanjing: Nangjing University of Posts and Telecommunications, 2012.

[6] Wenbin Gong, Zhongmin Gan. Optimal reservation guard channel in wireless system. Journal of applied sciences [J].2003,21(4): 427-430.
[7] Feixia Xue. Call admission control algorithm research in 3G LTE [D].Nanjing: Nanjing University of Posts and Telecommunications, 2009 .

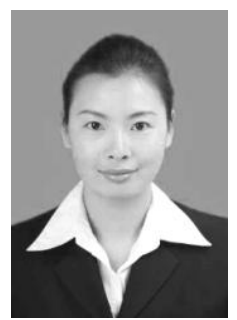

Han Haifang. was born in 1989. She is currently working toward the M.S. degrees in Xi'an University of Posts and Telecommunications. Her research interests include wireless broadband network.

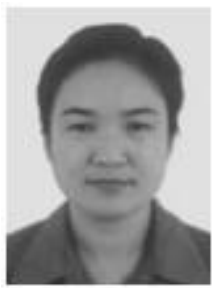

Zhao Jihong, was born in 1963, Ph D., professor of Xi'an University of Posts and Telecommunications and Nanjing University of Posts and Telecommunications. Her current research interests include wireless broadband network, mobile Internet, network management and control. 